\title{
Prodromus of vegetation of Yakutia
}

\author{
Paraskovia Gogoleva ${ }^{1 *}$, Mikhail Cherosov ${ }^{2}$, Svetlana Mironova ${ }^{1}$, Evdokia Burtseva ${ }^{1}$, \\ Nadezhda Sleptsova $^{1}$, Boris Pestryakov ${ }^{1}$, Praskovia Kharlampyeva ${ }^{1}$, Lyudmila Gavrilyeva $^{1}$, \\ Sargylana Poiseeva ${ }^{1}$, Nikolai Ermakov ${ }^{3}$, Mikhail Telyatnikov ${ }^{3}$, Nikolai Lashchinsky ${ }^{3}$, Elena \\ Troeva $^{2}$, Evgeny Nikolin ${ }^{2}$, and Viktoria Filippova ${ }^{2}$ \\ ${ }^{1}$ M.K. Ammosov North-Eastern Federal University, 58 Belinsky Str., Yakutsk, 677000, Russia \\ ${ }^{2}$ Institute for Biological Problems of Cryolithozone, Siberian Branch of the Russian Academy of \\ Sciences, 41 Lenin Ave., Yakutsk, 677980, Russia \\ ${ }^{3}$ Central Siberian Botanical Garden, Siberian Branch of the Russian Academy of Sciences, 101 \\ Zolotodolinskaya Str., Novosibirsk,463090, Russia
}

\begin{abstract}
A brief history of development of the Prodromus vegetation of
\end{abstract} Yakutia is given. The characteristic of classes is given in a table.

The use of Braun-Blanquet approach for classification of Yakutia vegetation was invigorated by Boris Mirkin, Professor of the Bashkir State University. In Yakutia, he guided the steps of a number of specialists of the Yakut State University in their postgraduate study of botany and landscapes. Under his scientific supervision, K.E. Kononov defended his doctoral dissertation on vegetation of the Lena River floodplain [1-5], E.I. Burtseva studied the meadows on saline soils of the Lena River valley [3, 4], P.A. Gogoleva studied the vegetation of alas landscapes of the Lena-Amga Interfluve [2, 4, $5,6,7,8]$, and S.I. Mironova also studied the alas vegetation of the Lena-Vilyuy Interfluve as well as recovery of vegetation of technogenic landscapes $[2,7,9,10,11]$.

The students and followers of Prof. Mirkin used Braun-Blanquet approach for classification of weed vegetation (N.P. Sleptsova) [12, 13], a number of northern taiga and tundra plant communities (B.N. Pestryakov) [14-16], vegetation of the Vilyuy River basin (S.I. Poiseeva) [17-19], ruderal vegetation (M.M. Cherosov) [8, 20, 21, 22], grazing land vegetation (L.D. Gavrilyeva) [10, 23, 24], forest and mountain vegetation (N.B. Ermakov, E.G. Nikolin) [25-29]. Recently, the works on tundra vegetation classification were conducted (M.Yu. Telyatnikov, N.N. Lashchinsky, E.I. Troeva) [30-33]. Aquatic vegetation classification is underway now (P.I. Kharlampyeva, V.A. Filippova) [34-35].

The long-term work yielded the Prodromus of vegetation of Yakutia. Its structure is represented now by the following units: 36 classes, 72 orders, 78 alliances, 216 associations, 112 subassociations, 63 variants, and 13 derivative and basal communities (Table).

As it is seen from the Table, riparian, meadow and anthropogenic vegetation is most properly studied. The higher rank units are given according to Ermakov (2012) [36].

\footnotetext{
*Corresponding author: sedum@mail.ru
} 
Table. Structure of the Prodromus of vegetation of Yakutia

\begin{tabular}{|c|c|c|c|c|c|}
\hline Class & Ord & All & Ass & Subass & Var/co \\
\hline 1 & 2 & 3 & 4 & 5 & 6 \\
\hline Lemnetea & 1 & 1 & 5 & & \\
\hline Potametea pectinati & 2 & 3 & 12 & & \\
\hline Utricularietea intermedio-minoris & 1 & 1 & 1 & & \\
\hline Littorelletea & 1 & 1 & 1 & & \\
\hline Phragmiti-Magno-Caricetea & 5 & 10 & 37 & 10 & 1 d.c. \\
\hline Thero-Salicornietea & 1 & 1 & 2 & & \\
\hline Bolboshoenetea maritimi & 1 & 2 & 3 & & \\
\hline Scorzonero-Juncetea gerardii & 2 & 3 & 9 & 18 & 5 \\
\hline Scheuchzerio-Caricetea nigrae & 3 & $5 / 1$ & 17 & 1 & 4 \\
\hline$?$ & 1 & 1 & 1 & 2 & \\
\hline Oxycocco-Sphagnetea & 1 & 1 & 1 & 2 & 1 \\
\hline Thlaspietea rotundifolii & 1 & 2 & 2 & 2 & \\
\hline Salicetea herbaceae & 1 & 1 & 4 & & 1 \\
\hline Carici rupestris-Kobresietea bellardii & 1 & 1 & 2 & 3 & \\
\hline Loiseleurio-Vaccinietea & 1 & 3 & 11 & 8 & 2 \\
\hline$?$ & 1 & 1 & 1 & & \\
\hline Juncetea trifidi & 1 & 1 & 1 & & \\
\hline Hylocomio-Salicetea glaucae & 1 & 1 & 1 & & 1 \\
\hline Molinio-Arrenatheretea & 2 & 3 & 7 & 5 & $7 / 1$ \\
\hline Calamagrostetea langsdorffii & 1 & 1 & 7 & & \\
\hline Cleistogenetea sguarrosae & 2 & $8 / 1$ & 17 & 13 & $2 / 1$ \\
\hline$?$ & 1 & 1 & 2 & 2 & \\
\hline Vaccinio-Piceetea & 5 & 6 & 20 & 4 & 4 \\
\hline Rhytidio-Laricetea sibiricae & 3 & 4 & 4 & & \\
\hline Polygono arenastri-Poetea annuae & 1 & 1 & 1 & 2 & \\
\hline Stellarietea mediae & 2 & 4 & 7 & 13 & 12 \\
\hline Artemisietea vulgaris & 2 & 3 & 5 & 9 & $13 / 3$ \\
\hline Polygono-Artemisietea austriacea & 1 & 1 & 4 & 2 & \\
\hline Epilobietea angustifolii & 1 & 1 & 2 & & 14 \\
\hline Bidentetea tripartiti & 1 & 1 & 2 & 2 & \\
\hline Puccinellio-Hordeetea jubati & 1 & 1 & 16 & 10 & 13 \\
\hline Matricario-Poetea arcticae & 1 & 1 & 6 & 2 & $2 / 1$ \\
\hline Plantaginetea majoris & 2 & 2 & 3 & & \\
\hline Salici-Betuletea nanae & 1 & 1 & & & $/ 1$ \\
\hline Salicetea purpureae & 1 & 1 & 2 & 4 & /7 b.c. \\
\hline Total & 72 & 78 & 216 & 112 & $63 / 13$ \\
\hline
\end{tabular}

\section{References}

1. K.E. Kononov, Ecology of meadow vegetation of the Lena River floodplain, Diss. (M, 1982)

2. K.E. Kononov, P.A. Gogoleva, S.I. Mironova, B.M. Mirkin, Classification of vegetation of the USSR my means of floristic criteria $(\mathrm{M}, 1986)$

3. B.M. Mirkin, E.I. Burtseva, K.E. Kononov, Biol. nauki, 9 (1984) 
4. B.M. Mirkin, K.E. Kononov, P.A. Gogoleva, E.I. Burtseva, L.G. Naumova, Folia Geobot. Phytotaxon, 27 (1992)

5. B.M. Mirkin, P.A. Gogoleva, K.E. Kononov, Folia Geobot. Phytotaxon, 20 (1983)

6. P.A. Gogoleva, Biol. Nauki, DEP, 246-83 (1983)

7. P.A. Gogoleva, K.E. Kononov, B.M. Mirkin, S.I. Mironova, Sintaxonomy and simphytosociology of alas vegetation of Central Yakutia (Irkutsk, 1987)

8. P.A. Gogoleva, M.M. Cherosov, S.G. Struchkova, E.D. Fedorova, Vestnik SVFU, 1(57) (2017)

9. S.I. Mironova, Ecology, 5 (1983)

10. S.I. Mironova, L.D. Gavrilyeva, Z.S. Pavlova, Nauch. Dokl. Bysch.Shkoly, 2485 (1990)

11. O.I. Sumina, S.I. Mironova, Polar Geography, 27(3) (2005)

12. N.P. Sleptsova, Bot. Zhurnal, 69(8) (1984)

13. N.P. Sleptsova, Weed vegetation of Central Yakutia (an experience of floristic classification application), Diss. (Tartu, 1985)

14. B.N. Pestryakov, P.A. Gogoleva, S.I. Mironova, DEP. AUISTI, 6289-B88 (1988)

15. B.N. Pestryakov, Ecological-phytosociological characterization of the vegetation in the Yana River valley (northeastern Yakutia) and synanthropization processes. Diss. (Ufa, 1991)

16. B.N. Pestryakov, P.A. Gogoleva, A.R. Ishbirdin, DEP. AUISTI, 3512-B92 (1992)

17. S.I. Poiseeva, Nauka i obrazovanie, 2 (2004)

18. S.I. Poiseeva, Problems of the study of vegetation cover of Yakutia (Yakutsk, Sakhapoligrafizdat, 2004)

19. S.I. Poiseeva, Fundamentalnye issledovaniya, 2 (2005)

20. P.A. Gogoleva, M.M. Cherosov, Z.S. Pavlova, B.M. Mirkin, DEP. AUISTI, B87 (1987)

21. M.M. Cherosov, Synanthropic vegetation of Yakutia (Yakutsk, 2005)

22. M.M. Cherosov, N.P. Sleptsova, S.I. Mironova, P.A. Gogoleva, Syntaxonomy of synanthropic vegetation of Yakutia (Yakutsk, 2005)

23. L.D. Gavrilyeva, Pasture digression and rational use of alas vegetation of the Lena-Amga Interfluve, Diss.(Yakutsk, 1998)

24. L.D. Gavrilyeva, Sib. Ecol. Zhurnal, 18(3) (2011)

25. N.B. Ermakov, Hemiboreal forests of continental Northern Asia (classification, ordination, coenoflora analysis), Diss. (Novosibirsk, 2001)

26. N.B. Ermakov, M.M. Cherosov, P.A. Gogoleva, Folia Geobot. Phytotaxon., 37 (2002)

27. N.B. Ermakov, M.M. Cherosov, Annali di Botanica, 5 (2005)

28. N.B. Ermakov, E.G. Nikolin, E.I. Troeva, M.M. Cherosov, Vestnik NGU, 7(4) (2009)

29. N.B. Ermakov, E.G. Nikolin, E.I. Troeva, M.M. Cherosov, Vestnik NGU, 8(3) (2010)

30. M.Yu. Telyatnikov, E.I. Troeva, P.A. Gogoleva, M.M. Cherosov, L.A. Pestryakova, S.A. Pristyazhnyuk, Flora of Asian Russia, 1(11) (2013) 
31. M.Yu. Telyatnikov, N.N. Laschinsky, E.I. Troeva, S.A. Pristyazhnyuk, P.A. Gogoleva, M.M. Cherosov, L.A. Pestryakova, Turczaninowia, 17 (2014)

32. M.Yu. Telyatnikov, E.I. Troeva, S.A. Pristyazhnyuk, P.A. Gogoleva, M.M. Cherosov, L.A. Pestryakova, Turczaninowia, 18(4) (2015)

33. M.Yu. Telyatnikov, E.I. Troeva, S.A. Pristyazhnyuk, M.M. Cherosov, Turczaninowia, 20(3) (2017)

34. P.I. Kharlampyeva, P.A. Gogoleva, L.A. Frolova, Scientific notes of Kazan University, 153(2) (2011)

35. V.A. Filippova, M.M. Cherosov, E.S. Neustroeva, I.I. Osipova, Byull. MOIP (2009)

36. N.B. Ermakov, Modern condition of basic concepts of vegetation science (Ufa, 2012) 